\title{
On Floer homology of the Gauss images of isoparametric hypersurfaces
}

\section{Yoshihiro Ohnita}

\begin{tabular}{|c|l|}
\hline Citation & OCAMI Preprint Series \\
\hline Issue Date & 2017 \\
\hline Type & Preprint \\
\hline Textversion & Author \\
\hline Rights & For personal use only. No other uses without permission. \\
\hline Relation & $\begin{array}{l}\text { This is a pre-print of an article published in Hermitian-Grassmannian } \\
\text { Submanifolds. The final authenticated version is available online at: } \\
\text { https://doi.org/10.1007/978-981-10-5556-0_20 } . ~\end{array}$ \\
\hline
\end{tabular}

From: Osaka City University Advanced Mathematical Institute http://www.sci.osaka-cu.ac.jp/OCAMI/publication/preprint/preprint.html 


\title{
On Floer homology of the Gauss images of isoparametric hypersurfaces
}

\author{
Yoshihiro Ohnita
}

\begin{abstract}
The Gauss images of isoparametric hypersurfaces in the unit standard sphere provide compact minimal (thus monotone) Lagrangian submanifolds embedded in complex hyperquadrics. Recently we used the Floer homology and the lifted Floer homology for monotone Lagrangian submanifolds in order to study their Hamiltonian non-displaceability in our recent joint paper with Hiroshi Iriyeh, Hui Ma and Reiko Miyaoka. In this note we will explain the spectral sequences for the Floer homology and the lifted Floer homology of monotone Lagrangian submanifolds and their applications to the Gauss images of isoparametric hypersurfaces. They are the main technical part in our joint work. Moreover we will suggest some related open problems for further research.
\end{abstract}

\section{Introduction}

Let $N^{n}$ be an isoparametric hypersurface in the unit standard sphere $S^{n+1} \subset \mathbb{R}^{n+2}$. By the structure theory of isoparametric hypersurfaces (see [16]), $N^{n}$ is nothing but a hypersurface of constant principal curvatures and if we denote by $g$ the distinct number of principal curvatures of $N^{n}$ and by $m_{1}, m_{2}, \cdots, m_{g}$ their multiplicities, then we know $m_{i}=m_{i+2}(i \bmod g)$. Moreover, it is known that $N^{n}$ can be extended to a compact oriented hypersurface embedded in $S^{n+1}$.

We know that isoparametric hypersurfaces in $S^{n+1}$ provide a nice class of Lagrangian submanifolds in the complex hyperquadric $Q_{n}(\mathbb{C})$. Note that a complex hyperquadric $Q_{n}(\mathbb{C})$ can be identified with a real Grassmann manifold $\widetilde{G r} 2\left(\mathbb{R}^{n+2}\right)$ of oriented 2-dimensional vector subspaces of $\mathbb{R}^{n+2}$ in the standard way:

Yoshihiro Ohnita

Osaka City University Advanced Mathematical Institute, 3-3-138 Sugimoto, Sumiyoshi-ku,Osaka 558-8585 JAPAN, e-mail: ohnita@sci.osaka-cu.ac.jp 


$$
\begin{aligned}
Q_{n}(\mathbb{C}) & \cong \widetilde{G r} r_{2}\left(\mathbb{R}^{n+2}\right) \cong S O(n+2) /(S O(2) \times S O(n)) \\
{[\mathbf{a}+\sqrt{-1} \mathbf{b}] } & \leftrightarrow \quad \mathbf{a} \wedge \mathbf{b} \leftrightarrow[\mathbf{a}, \mathbf{b}, \cdots](S O(2) \times S O(n))
\end{aligned}
$$

It is an irreducible compact Hermitian symmetric space of rank 2 for $n \geq 3$. If $n=2$, then $Q_{2}(\mathbb{C}) \cong S^{2} \times S^{2}$, and if $n=1$, then $Q_{1}(\mathbb{C}) \cong \mathbb{C} P^{1}$. We denote by $\omega_{\text {std }}$ the standard Kähler form of $Q_{n}(\mathbb{C})$.

In general the Gauss map $\mathscr{G}$ of an oriented hypersurface $N^{n}$ immersed in the unit standard sphere $S^{n+1}$ is defined by

$$
\mathscr{G}: N^{n} \ni p \longmapsto[\mathbf{x}(p)+\sqrt{-1} \mathbf{n}(p)] \in Q_{n}(\mathbb{C}) .
$$

Then we know that $\mathscr{G}: N^{n} \rightarrow\left(Q_{n}(\mathbb{C}), \omega_{\text {std }}\right)$ is always a Lagrangian immersion. Palmer ([23]) gave a formula expressing the mean curvature form of $\mathscr{G}$ in terms of principal curvatures of $N^{n}$ in $S^{n+1}$ and from this formula he observed that if $N^{n}$ has constant principal curvatures, then the Gauss map $\mathscr{G}: N^{n} \rightarrow\left(Q_{n}(\mathbb{C}), \omega_{\text {std }}\right)$ is a minimal Lagrangian immersion. Note that the Gauss map is not necessary an embedding into $Q_{n}(\mathbb{C})$.

For each isoparametric hypersurface $N^{n}$ in $S^{n+1}$ the image of the Gauss map, which is called the Gauss image $\mathscr{G}\left(N^{n}\right)$, has the following nice properties.

Theorem 1 ([12], [14], [21]). Suppose that $N^{n}$ is a compact oriented isoparametric hypersurface embedded in $S^{n+1}$ with $g$ distinct principal curvatures and multiplicities $\left(m_{1}, m_{2}\right)$. Then the Gauss image $\mathscr{G}\left(N^{n}\right)$ has the following properties:

(1) The Gauss image $L^{n}=\mathscr{G}\left(N^{n}\right)$ is a compact smooth minimal Lagrangian submanifold embedded in $Q_{n}(\mathbb{C})$.

(2) The Gauss map $\mathscr{G}$ into the Gauss image $L^{n}=\mathscr{G}\left(N^{n}\right)$ gives a covering map $\mathscr{G}: N^{n} \rightarrow L^{n}=\mathscr{G}\left(N^{n}\right) \cong N^{n} / \mathbb{Z}_{g}$ with the covering transformation group $\mathbb{Z}_{g}$.

(3) The Gauss image $L^{n}=\mathscr{G}\left(N^{n}\right)$ is monotone in $Q_{n}(\mathbb{C})$ and its minimal Maslov number $\Sigma_{L}$ is given by

$$
\Sigma_{L}=\frac{2 n}{g}= \begin{cases}m_{1}+m_{2}, & \text { if } g \text { is even } \\ 2 m_{1}, & \text { if } g \text { is odd }\end{cases}
$$

The Gauss image $\mathscr{G}\left(N^{n}\right)$ is orientable (resp. non-orientable) if and only if $2 n / g$ is even (resp. odd).

It is a natural and interesting problem to study the properties of the Guass image of isoparametric hypersurfaces in $S^{n+1}$ as Lagrangian submanifolds embedded in $Q_{n}(\mathbb{C})$ ([23], [12], [13], [14], [15]).

Recently in our recent joint paper with Hiroshi Iriyeh, Hui Ma and Reiko Miyaoka ([11]) in order to study their Hamiltonian non-displaceability we used the Floer homology and the lifted Floer homology for monotone Lagrangian submanifolds. In this note we will explain the construction of the Floer homology and the spectral sequences for monotone Lagrangian submanifolds, and also their lifted Floer homology (Floer, Y.-G. Oh, Biran, Damian), and their applications to the Gauss images of isoparametric hypersurfaces. They are the main technical part in 
our joint work. The ideas to use the spectral sequence and the lifted Floer homology in this case are due to H. Iriyeh ([9]) . Moreover we will suggest some related open problems for further research.

Throughout this article any manifold is smooth and connected.

\section{Floer homology of monotone Lagrangian submanifolds}

Let $(M, \omega)$ be a symplectic manifold. If a diffeomorphism $\phi$ of $M$ is given by $\phi=\phi_{1}$ for some time-dependent Hamiltonian $H_{t}(t \in[0,1])$ and an isotopy $\phi_{t}: M \rightarrow$ $M(t \in[0,1])$ of $M$ satisfying the Hamiltonian equation

$$
\frac{d \phi_{t}(x)}{d t}=\left(X_{H_{t}}\right)_{\phi_{t}(x)} \quad \text { and } \quad \phi_{0}(x)=x \quad(x \in M),
$$

then $\phi$ is called a Hamiltonian diffeomorphism of $(M, \omega)$ and $\left\{\phi_{t}\right\}_{t \in[0,1]}$ is called a Hamiltonian isotopy of $(M, \omega)$. Here $X_{H_{t}}$ denotes a Hamiltonian vector field corresponding to a Hamiltonian $H_{t}$ with respect to $\omega$. Let $\operatorname{Haml}(M, \omega)$ denote a set of all Hamiltonian diffeomorphisms of $(M, \omega)$. Then $\operatorname{Haml}(M, \omega)$ is a subgroup of the identity component $\operatorname{Symp}^{0}(M, \omega)$ of the symplectic diffeomorphism group of $(M, \omega)$. A Lagrangian submanifold $L$ in $M$ is called Hamiltonian non-displaceable if $L \cap \phi(L) \neq \emptyset$ for any $\phi \in \operatorname{Haml}(M, \omega)$, and it is called Hamiltonian displaceable otherwise. It is well-known that in the 2-dimensional standard sphere a small circle is Hamiltonian displaceable but a great circle is Hamiltonian non-displaceable. Then the following is one of elementary questions in symplectic geometry:

Question. What Lagrangian submanifolds are Hamiltonian non-displaceable?

Let $(M, \omega)$ be a closed symplectic manifold and $L$ be a closed (i.e. compact without boundary) Lagrangian submanifold embedded in $M$. Let $\phi \in \operatorname{Haml}(M, \omega)$ such that $L$ and $\phi(L)$ intersects transversally (denoted by the symbol $L \pitchfork \phi(L)$ ). Let $\left(\phi_{t}\right)_{t \in[0,1]}$ be a Hamiltonian isotopy of $(M, \omega)$ with $\phi_{1}=\phi$ and set $L_{t}:=\phi_{t}(L)(t \in$ $[0,1])$.

Choose an almost complex structure $J$ on $M$ compatible with $\omega$ (i.e. $\omega(\cdot, J \cdot)$ is a Riemannian metric on $M$ ). Define

$$
\begin{aligned}
& \mathscr{M}\left(L_{0}, L_{1}\right):=\left\{u \in C^{\infty}(\mathbb{R} \times[0,1]) \mid\right. \frac{\partial u}{\partial \tau}+J_{t} \frac{\partial u}{\partial t}=0, \\
& u(\tau, 0) \subset L, u(\tau, 1) \subset \phi_{1}(L), \\
&\left.E(u):=\frac{1}{2} \int_{\mathbb{R} \times[0,1]}\|d u\|^{2} d \tau d t<\infty\right\}
\end{aligned}
$$

For $x, y \in L_{0} \cap L_{1}$, set

$$
\mathscr{M}(x, y):=\left\{u \in \mathscr{M}\left(L_{0}, L_{1}\right) \mid \lim _{\tau \rightarrow-\infty} u(\tau, \cdot)=x, \lim _{\tau \rightarrow \infty} u(\tau, \cdot)=y\right\} .
$$


Floer showed

$$
\mathscr{M}\left(L_{0}, L_{1}\right)=\bigcup_{x, y \in L_{0} \cap L_{1}} \mathscr{M}(x, y)
$$

For a generic choice $J$, a neighborhood of each $u \in \mathscr{M}(x, y)$ is a smooth manifold of finite dimension equal to $\mu_{u}(x, y)=$ the Maslov-Virerbo index of $u$.

Let $\widehat{\mathscr{M}}(x, y):=\mathscr{M}(x, y) / \mathbb{R}$ be the moduli space of holomorphic strips joining from $x$ to $y$ modulo translations with respect to $\tau$. Then note that for such a choice $J$, a neighborhood of each $[u] \in \widehat{\mathscr{M}}(x, y)$ is a smooth manifold of finite dimension equal to $\mu_{u}(x, y)-1$. Denote by $\widehat{\mathscr{M}}^{0}(x, y)$ (resp. $\left.\widehat{\mathscr{M}}^{\mathrm{I}}(x, y)\right)$ the 0 -dimensional (resp. 1-dimensional) component of $\widehat{\mathscr{M}}(x, y)$.

For a given Lagrangian submanifold $L$ of a symplectic manifold $(M, \omega)$, two kinds of group homomorphisms $I_{\mu, L}: \pi_{2}(M, L) \rightarrow \mathbb{Z}$ and $I_{\omega, L}: \pi_{2}(M, L) \rightarrow \mathbb{R}$ are defined as follows: For a smooth map $u:\left(D^{2}, \partial D^{2}\right) \rightarrow(M, L)$ belonging to $A \in$ $\pi_{2}(M, L)$, choose a trivialization of the pull-back bundle $u^{-1} T M \cong D^{2} \times \mathbb{C}^{n}$ as a symplectic vector bundle, which is unique up to the homotopy. This gives a smooth map $\tilde{u}$ from $S^{1}=\partial D^{2}$ to $\Lambda\left(\mathbb{C}^{n}\right)$. Here $\Lambda\left(\mathbb{C}^{n}\right)$ denotes a Grassmann manifold of Lagrangian vector subspaces of $\mathbb{C}^{n}$. Using the Moslov class $\mu \in H^{1}\left(\Lambda\left(\mathbb{C}^{n}\right), \mathbb{Z}\right)$, we can define $I_{\mu, L}(A):=\mu(\tilde{u})$. Next $I_{\omega, L}$ is defined by $I_{\omega, L}(A):=\int_{D^{2}} u^{*} \omega . I_{\mu, L}$ is invariant under symplectic diffeomorphisms and $I_{\omega, L}$ is invariant under Hamiltonian diffeomorphisms but not under symplectic diffeomorphisms.

A Lagrangian submanifold $L$ is called monotone if $I_{\mu, L}=\lambda I_{\omega, L}$ for some constant $\lambda>0$. We denote by $\Sigma_{L} \in \mathbb{Z}_{+}$a positive generator of an additive subgroup $\operatorname{Im}\left(I_{\mu, L}\right) \subset \mathbb{Z}$ and $\Sigma_{L}$ is called the minimal Maslov number of $L$. It is known that any compact minimal Lagrangian submanifold in an Einstein-Kähler manifold of positive Einstein constant is monotone ([22]).

The compactness and compactification of 0 -dimensional and 1-dimensional moduli spaces of holomorphic strips are due to Gromov, Floer, Y.-G. Oh.

Theorem 2 (Compactness). Suppose that $L$ is compact and monotone with $\Sigma_{L} \geq 2$. Let $x, y \in L_{0} \cap L_{1}$ and $A>0$. Let $\left\{u_{\alpha}\right\} \subset \mathscr{M}(x, y)$ be a sequence with constant index $\mu\left(u_{\alpha}\right)=\mu_{0} \leq 2$ and with $E\left(u_{\alpha}\right)<A$. Then there exists a finite subset $\left\{z_{0}, \cdots, z_{k}\right\} \subset$ $L_{0} \cap L_{1}$ with $z_{0}=x$ and $z_{k}=y$, some $u^{i} \in \mathscr{M}\left(z_{i-1}, z_{i}\right)$ for $i=1, \cdots, k$, and a sequence of real numbers $\left\{\sigma_{\alpha}^{i}\right\}_{\alpha}$ for $i=1, \cdots, k$, such that for each $i=1, \cdots, k$ the sequence $\left\{u_{\alpha}\left(\tau+\sigma_{\alpha}^{i}, t\right)\right\}_{\alpha}$ converges to $u^{i}(\tau, t)$ in $C_{\mathrm{loc}}^{\infty}$, and moreover $\sum_{i=1}^{k} \mu\left(u^{k}\right)=\mu_{0}$.

If $\mu_{0}=1$, then we have $k=1$ and $\left\{u_{\alpha}\left(\tau+\sigma_{\alpha}, t\right)\right\}_{\alpha}$ converges to $u^{1}(\tau, t)$ in $C_{\text {loc }}^{\infty}$, because of $\mu\left(u^{k}\right) \geq 1$. It implies that the 0 -dimensional component of the moduli space $\mathscr{L}^{0}(x, y)$ is compact and thus a finite set.

Theorem 3 (Compactification). Suppose that L is compact and monotone with $\Sigma_{L} \geq 3$. Then

$$
\widehat{\mathscr{M}^{1}}(x, y):=\widehat{\mathscr{M}}^{\mathrm{I}}(x, y) \cup \bigcup_{z \in L_{0} \cap L_{1}}\left(\widehat{\mathscr{M}}^{0}(x, z) \times \widehat{\mathscr{M}}^{0}(z, y)\right)
$$


is a compact 1-dimensional smooth manifold whose boundary is

$$
\bigcup_{z \in L_{0} \cap L_{1}}\left(\widehat{\mathscr{M}}^{0}(x, z) \times \widehat{\mathscr{M}}^{0}(z, y)\right) .
$$

Let

$$
C F(L, \phi):=\bigoplus_{x \in L \cap \phi(L)} \mathbb{Z}_{2} x
$$

denote a free $\mathbb{Z}_{2}$-module over all intersection points of $L$ and $\phi(L)$ where $\phi \in$ $\operatorname{Haml}(M, \omega)$. By Theorem 2, since $\widehat{\mathscr{M}}^{0}(x, y)$ is finite, we can define $n(x, y):=$ $\sharp \widehat{\mathscr{L}}^{0}(x, y) \bmod 2$. Then the Floer boudary operator $\partial_{J}$ is defined by

$$
\partial_{J}(x):=\sum_{y \in L \cap \phi(L)} n(x, y) y \quad(y \in L \cap \phi(L)) .
$$

Assume that $L$ is monotone with minimal Maslov number $\Sigma_{L} \geq 2$. Then we know that $\partial_{J} \circ \partial_{J}=0$, by Theorem 3 if $\Sigma_{L} \geq 3$, or by [18] if $\Sigma_{L} \geq 2$. The homology $H_{*}\left(C F(L, \phi), \partial_{J}\right)$ for the chain complex $\left(C F(L, \phi), \partial_{J}\right)$ does not depend on the choice of $J \in \mathscr{J}_{\text {reg }}(M, \omega)$ and $\phi \in \operatorname{Haml}(M, \omega)$ (Floer, Y. G. Oh). The Floer homology of $L$ is defined by

$$
H F(L):=H_{*}\left(C F(L, \phi), \partial_{J}\right) .
$$

Now fix an element $x_{0} \in L \cap \phi(L)$. We define a grading of $x \in L \cap \phi(L)$ by $\mu_{u}\left(x, x_{0}\right) \bmod \Sigma_{L}$. Here we use a fact that $\mu_{u}\left(x, x_{0}\right)-\mu_{v}\left(x, x_{0}\right)$ is a multiple of $\Sigma_{L}$ for arbitrary smooth maps $u, v:[0,1] \times[0,1] \rightarrow M$ with $u(\tau, 0), v(\tau, 0) \in L$, $u(\tau, 1), v(\tau, 1) \in \phi(L)$ and $u(\tau, 0), v(\tau, 0) \in L, u(\tau, 1), v(\tau, 1) \in \phi(L)$ ([17], p.973, Lemma 4.7). Thus the Floer complex $C F(L, \phi)$ has a $\mathbb{Z} / \Sigma_{L}$-grading, which depends on a choice of a base intersection point $x_{0} \in L \cap \phi(L)$. Denote this grading by

$$
C F(L, \phi)=\bigoplus_{i=0}^{\Sigma_{L}-1} C F_{i \bmod \Sigma_{L}}\left(L, \phi, x_{0}\right)
$$

where

$$
C F_{i \bmod \Sigma_{L}}\left(L, \phi, x_{0}\right):=\bigoplus_{x \in L \cap \phi(L), \mu_{u}\left(x, x_{0}\right) \equiv i \bmod \Sigma_{L}} \mathbb{Z}_{2} x .
$$

Let $x, y \in L \cap \phi(L)$. For $u \in \widehat{\mathscr{M}}^{0}(x, y)$, since by a composition formula ([5], p.406)

$$
\mu_{u v}\left(x, x_{0}\right)=\mu_{u}(x, y)+\mu_{v}\left(y, x_{0}\right)
$$

where $u v$ is a composition of $u$ and $v$, we have

$$
\mu_{v}\left(y, x_{0}\right)=\mu_{u v}\left(x, x_{0}\right)-\mu_{u}(x, y)=\mu_{u v}\left(x, x_{0}\right)-1 .
$$

Thus the Floer boudary operator decreases the grading by 1: 


$$
\partial_{J}: C F_{i \bmod \Sigma_{L}}\left(L, \phi, x_{0}\right) \longrightarrow C F_{i-1 \bmod \Sigma_{L}}\left(L, \phi, x_{0}\right) .
$$

Hence it induces a $\mathbb{Z} / \Sigma_{L}$-grading of the Floer homology as

$$
H F(L, \phi)=\bigoplus_{i=0}^{\Sigma_{L}-1} H F_{i \bmod \Sigma_{L}}\left(L, \phi \cdot x_{0}\right) .
$$

The grading of the Floer homology of $L$ is also preserved under any Hamiltonian diffeomorphism of $(M, \omega)$ (Floer, Y.-G. Oh [17]).

\section{Spectral sequence for Floer homology and lifted Floer homology}

Let $\left(M^{2 n}, \omega\right)$ be a compact symplectic manifold of dimension $2 n$. Let $L$ be a compact Lagrangian submanifold embedded in $M$. For a Hamiltonian isotopy $\left(\phi_{t}\right)_{t \in[0,1]}$ of $M$ with $L \pitchfork \phi(L)$, set $\phi=\phi_{1} \in \operatorname{Haml}(M, \omega)$ and $L^{\prime}=\phi(L)=\phi_{1}(L)$.

Consider a Morse-Smale function $f$ on $L$ and a particular Hamiltonian isotopy $\left(\phi_{t}\right)_{t \in[0,1]}$ which maps $L$ to $\phi_{t}(L)=d(t f)(L) \subset \mathscr{W} \subset M$. Here $\mathscr{W}$ is a Weinstein neighborhood of $L$ in $M$ which is symplectically diffeomorphic to a tubular neighborhood of the zero section of $T^{*} L$. In this situation note that $L \cap \phi_{1}(L)$ coincide with the critical point set $\operatorname{Crit}(f)$ of $f$ on $L$. We may assume that $f$ has exactly one relative minimum point $x_{0}$ on $L$. We choose $x_{0}$ as a base intersection point of $L \cap \phi(L)$. Let $\operatorname{Crit}(f)$ denote the set of all critical points of $f$ and $\mathrm{Crit}_{k}(f)$ the subset of all critical points of $f$ with index $\operatorname{ind}(f)_{x}=k$. Denote by $\left(C_{*}^{f}, \partial^{f}\right)$ the Morse complex for $f$, where

$$
C_{*}^{f}=\bigoplus_{k=0}^{n} C_{k}^{f}, \quad \text { where } \quad C_{k}^{f}=\bigoplus_{x \in \operatorname{Crit}_{k}(f)} \mathbb{Z}_{2} x
$$

and $\partial^{f}: C_{k}^{f} \rightarrow C_{k-1}^{f}$ is the Morse boudary operator of $f$. In this situation note that the Maslov-Viterbo index of $u$ coincides with the Morse index of $f$ : For each $x \in$ $L \cap \phi(L)=\operatorname{Crit}(f)$ and a smooth map $v:[0,1] \times[0,1] \rightarrow \mathscr{W} \subset M$ with $v(\tau, 0) \in L$, $v(\tau, 1) \in \phi(L), v(0, t)=x$ and $v(1, t)=y$,

$$
\mu_{v}\left(x, x_{0}\right)=\operatorname{ind}(f)_{x}-\operatorname{ind}(f)_{x_{0}}=\operatorname{ind}(f)_{x}
$$

([24, p. 370, Proiposition 5], [20, p. 318, Lemma 4]). Thus by the definition of a grading of $C F(L, \phi)$ we have

$$
C F_{i \bmod \Sigma_{L}}\left(L, \phi, x_{0}\right)=\bigoplus_{k \in \mathbb{Z}, k \equiv i \bmod \Sigma_{L}} C_{k}^{f}=\bigoplus_{\ell \in \mathbb{Z}} C_{i+\ell \Sigma_{L}}^{f} .
$$

We follow the argument of [20], For a sufficiently small $t$ we can take a disk $w$ : $\left(D^{2}, \partial D^{2}\right) \rightarrow(M, L)$ with $I_{\omega, L}([w])>0$ by gluing $u \in \widehat{\mathscr{M}}^{0}(x, y)$ with $\operatorname{Im} u \not \subset \mathscr{W}$ to a 
thin strip $v$ between $\phi_{t}(L)$ and $L$ connecting $y$ and $x$. By the monotonicity of $L$ note that $I_{\mu, L}([w])=\lambda I_{\omega, L}([w])>0$, and thus $I_{\mu, L}([w])=\ell \Sigma_{L}$ for some $\ell \in \mathbb{N}$. Thus we have

$$
\begin{aligned}
0<I_{\mu, L}([w]) & =\mu_{u}(x, y)-\mu_{u^{\prime}}(x, y) \\
& =1-\left(\operatorname{ind}(f)_{x}-\operatorname{ind}(f)_{y}\right) \\
& =1-\operatorname{ind}(f)_{x}+\operatorname{ind}(f)_{y} \leq n+1 .
\end{aligned}
$$

Hence we obtain $1 \leq \ell \leq \frac{n+1}{\Sigma_{L}}$ and $\operatorname{ind}(f)_{y}=\operatorname{ind}(f)_{x}-1+\ell \Sigma_{L}$. Set $v:=\left[\frac{n+1}{\Sigma_{L}}\right]$. Since we see that $\partial_{J}\left(C_{k}^{f}\right) \subset \bigoplus_{\ell=0}^{v} C_{k-1+\ell \Sigma_{L}}^{f}$, the Floer boudary operator $\partial_{J}$ can be decomposed as

$$
\partial_{J}=\partial_{0}+\partial_{1}+\cdots+\partial_{\nu}
$$

where $\partial_{\ell}: C_{*}^{f} \rightarrow C_{*-1+\ell \Sigma_{L}}^{f}(\ell=1, \cdots, v)$. Here note that $\partial_{0}$ counts small isolated Floer trajectries ( $J$-holomorphic strips) contained in a Weinstein neighborhood of $L$ and it coincides with the Morse boudary operator $\partial^{f}$ of $f$ (local Floer homology [20]). The operator $\partial_{1}+\cdots+\partial_{v}$ expresses a contribution of large isolated Floer trajectries.

Y.-G. Oh ([20]) and Biran ([1]) showed the existence of a spectral sequence $\left\{E_{r}^{p, q}, d_{r}\right\}$ converging towards the Floer homology. Such a spectral sequence was constructed by Biran in the following way ([1]).

Let $A:=\mathbb{Z}_{2}\left[T, T^{-1}\right]=\bigoplus_{k \in \mathbb{Z}} A^{k \Sigma_{L}}$ be the algebra of Laurent polynomials over $\mathbb{Z}_{2}$ with the variable $T$. Here define $\operatorname{deg}(T)=\Sigma_{L}$ and $A^{k \Sigma_{L}}:=\mathbb{Z}_{2} T^{k}$. Now let

$$
\widetilde{C}:=C^{f} \otimes A=\bigoplus_{k \in \mathbb{Z}} C^{f} \otimes A^{k \Sigma_{L}}=\bigoplus_{i \in \mathbb{Z}} \widetilde{C}_{i}
$$

where

$$
\widetilde{C}_{i}:=\bigoplus_{k \in \mathbb{Z}} C_{i-k \Sigma_{L}}^{f} \otimes A^{k \Sigma_{L}}=\bigoplus_{k \in \mathbb{Z}, \frac{i-n}{\Sigma_{L}} \leq k \leq \frac{i}{\Sigma_{L}}} C_{i-k \Sigma_{L}}^{f} \otimes A^{k \Sigma_{L}} .
$$

Define $\widetilde{\partial}_{J}: \widetilde{C}_{*} \rightarrow \widetilde{C}_{*-1}$ by

$$
\widetilde{\partial}_{J}:=\partial_{0} \otimes 1+\partial_{1} \otimes \tau+\cdots+\partial_{v} \otimes \tau^{v},
$$

where each $\tau^{\ell}: A^{*} \rightarrow A^{*-\ell \Sigma_{L}}$ is the multiplication by $T^{-\ell}$. Then we know that $\widetilde{\partial}_{J}$ : $\widetilde{C} \rightarrow \widetilde{C}$ satisfies $\widetilde{\partial}_{J} \circ \widetilde{\partial}_{J}=0$, that is, $(\widetilde{C}, \widetilde{\partial})$ is a chain complex. Moreover, as vector spaces over $\mathbb{Z}_{2}$, we obtain

$$
H F_{i \bmod \Sigma_{L}}(L) \cong H_{i}\left(\widetilde{C}, \widetilde{\partial}_{J}\right)=\frac{\operatorname{Ker}\left(\widetilde{\partial}_{J}: \widetilde{C}_{i} \rightarrow \widetilde{C}_{i-1}\right)}{\operatorname{Im}\left(\widetilde{\partial}_{J}: \widetilde{C}_{i+1} \rightarrow \widetilde{C}_{i}\right)} \quad(\forall i \in \mathbb{Z})
$$

and

$$
H\left(\widetilde{C}, \widetilde{\partial}_{J}\right)=\frac{\operatorname{Ker}\left(\widetilde{\partial}_{J}: \widetilde{C} \rightarrow \widetilde{C}\right)}{\operatorname{Im}\left(\widetilde{\partial}_{J}: \widetilde{C} \rightarrow \widetilde{C}\right)}=\bigoplus_{i \in \mathbb{Z}} H_{i}\left(\widetilde{C}, \widetilde{\partial}_{J}\right) \cong H F(L) \otimes_{\mathbb{Z}_{2}} A
$$


For each $p \in \mathbb{Z}$, set

$$
A_{p}:=\bigoplus_{k \leq p} A^{k \Sigma_{L}} \subset A
$$

and define

$$
F^{p} \widetilde{C}:=C^{f} \otimes A_{p} .
$$

Then we have an increasing filtration on $\widetilde{C}=\bigcup_{p \in \mathbb{Z}} F^{p} \widetilde{C}$ :

$$
\cdots \subset F^{-1} \widetilde{C} \subset F^{0} \widetilde{C} \subset \cdots \subset F^{p} \widetilde{C} \subset F^{p+1} \widetilde{C} \subset \cdots
$$

For each $p, l \in \mathbb{Z}$, define

$$
F^{p} \widetilde{C}_{l}:=F^{p} \widetilde{C} \cap \widetilde{C}_{l}=\bigoplus_{k \leq p} C_{l-k \Sigma_{L}}^{f} \otimes A^{k \Sigma_{L}}
$$

Then for each $l \in \mathbb{Z}$ we have the increasing filtration on $\widetilde{C}_{l}=\bigcup_{p \in \mathbb{Z}} F^{p} \widetilde{C}_{l}$

$$
\cdots \subset F^{-1} \widetilde{C}_{l} \subset F^{0} \widetilde{C}_{l} \subset \cdots \subset F^{p} \widetilde{C}_{l} \subset F^{p+1} \widetilde{C}_{l} \subset \cdots
$$

which satisfies $F^{p} \widetilde{C}_{l}=\{0\}$ for any $p<\frac{l-n}{\Sigma_{L}}$ and $F^{p} \widetilde{C}_{l}=\widetilde{C}_{l}$ for any $p \geq \frac{l}{\Sigma_{L}}$. And for each $p \in \mathbb{Z}, F^{p} \widetilde{C}$ has a grading

$$
F^{p} \widetilde{C}=\bigoplus_{l \in \mathbb{Z}} F^{p} \widetilde{C}_{l}
$$

For each $p \in \mathbb{Z}$ with $p>\frac{i}{\Sigma_{L}}$

$$
\begin{aligned}
H_{i}\left(F^{p} \widetilde{C}, \widetilde{\partial}_{J}\right) & =\frac{\operatorname{Ker}\left(\widetilde{\partial}_{J}: F^{p} \widetilde{C}_{i} \rightarrow F^{p} \widetilde{C}_{i-1}\right)}{\operatorname{Im}\left(\widetilde{\partial}_{J}: F^{p} \widetilde{C}_{i+1} \rightarrow F^{p} \widetilde{C}_{i}\right)} \\
& =\frac{\operatorname{Ker}\left(\widetilde{\partial}_{J}: F \widetilde{C}_{i} \rightarrow F \widetilde{C}_{i-1}\right)}{\operatorname{Im}\left(\widetilde{\partial}_{J}: F \widetilde{C}_{i+1} \rightarrow F \widetilde{C}_{i}\right)}=H_{i}\left(\widetilde{C}, \widetilde{\partial}_{J}\right) \cong H F_{i \bmod \Sigma_{L}}(L)
\end{aligned}
$$

and 


$$
\begin{aligned}
& H\left(F^{p} \widetilde{C}, \widetilde{\partial}_{J}\right)=\frac{\operatorname{Ker}\left(\widetilde{\partial}_{J}: F^{p} \widetilde{C} \rightarrow F^{p} \widetilde{C}\right)}{\operatorname{Im}\left(\widetilde{\partial}_{J}: F^{p} \widetilde{C} \rightarrow F^{p} \widetilde{C}\right)} \\
& =\bigoplus_{i \in \mathbb{Z}} H_{i}\left(F^{p} \widetilde{C}, \widetilde{\partial}_{J}\right) \\
& =\bigoplus_{i<p \Sigma_{L}} H_{i}\left(F^{p} \widetilde{C}, \widetilde{\partial}_{J}\right) \oplus \bigoplus_{p \Sigma_{L} \leq i} H_{i}\left(F^{p} \widetilde{C}, \widetilde{\partial_{J}}\right) \\
& =\bigoplus_{i<p \Sigma_{L}} H_{i}\left(\widetilde{C}, \widetilde{\partial}_{J}\right) \oplus \bigoplus_{p \Sigma_{L} \leq i \leq p \Sigma_{L}+n} H_{i}\left(F^{p} \widetilde{C}, \widetilde{\partial}_{J}\right) \\
& \cong \bigoplus_{i<p \Sigma_{L}} H F_{i \bmod \Sigma_{L}}(L) \oplus \bigoplus_{p \Sigma_{L} \leq i \leq p \Sigma_{L}+n} H_{i}\left(F^{p} \widetilde{C}, \widetilde{\partial}_{J}\right) .
\end{aligned}
$$

For each $p \in \mathbb{Z}$, since $\left(F^{p} \tilde{C}=\bigoplus_{l \in \mathbb{Z}} F^{p} \widetilde{C}_{l}, \widetilde{\partial}_{J}\right)$ is a graded filtered complex with filtration $\left\{\cdots \subset F^{p-2} \widetilde{C} \subset F^{p-1} \widetilde{C} \subset F^{p} \widetilde{C}\right\}$ and for each $l \in \mathbb{Z}$ the filtration $\{\cdots \subset$ $\left.F^{p-2} \widetilde{C}_{l} \subset F^{p-1} \widetilde{C}_{l} \subset F^{p} \widetilde{C}_{l}\right\}$ has finite length, there exists a spectral sequence which converges to $H_{*}\left(F^{p} \tilde{C}, \widetilde{\partial_{J}}\right)$ (cf. Bott-Tu [2, p.160, Theorem 14.6]). The following spectral sequence $\left\{E_{r}^{p, q}, d_{r}\right\}$ converging to $H F(L)$ was constructed by Biran [1]:

(1)

$$
E_{0}^{p, q}=C_{p+q-p \Sigma_{L}}^{f} \otimes A^{p \Sigma_{L}}, \quad d_{0}=\left[\partial_{0}\right] \otimes 1,
$$

$$
E_{1}^{p, q}=H_{p+q-p \Sigma_{L}}\left(L, \mathbb{Z}_{2}\right) \otimes A^{p \Sigma_{L}}, \quad d_{1}=\left[\partial_{1}\right] \otimes T^{-\Sigma_{L}},
$$

where

$$
\left[\partial_{1}\right]: H_{p+q-p \Sigma_{L}}\left(L ; \mathbb{Z}_{2}\right) \rightarrow H_{p+q-1-(p-1) \Sigma_{L}}\left(L ; \mathbb{Z}_{2}\right)
$$

is induced by the operator $\partial_{1}$.

(3) For each $r \geq 1, E_{r}^{p, q}$ has the form $E_{r}^{p, q}=V_{r}^{p, q} \otimes A^{p \Sigma_{L}}$ and

$$
d_{r}=\delta_{r} \otimes T^{-r \Sigma_{L}}: E_{r}^{p, q} \rightarrow E_{r}^{p-r, q+r-1},
$$

where each $V_{r}^{p, q}$ is a vector space over $\mathbb{Z}_{2}$ and $\delta_{r}: V_{r}^{p, q} \rightarrow V_{r}^{p-r, q+r-1}$ are homomorphisms defined for all $p, q$ satisfying $\delta_{r} \circ \delta_{r}=0$. Moreover, it holds

$$
V_{r+1}^{p, q}=\frac{\operatorname{Ker}\left(\delta_{r}: V_{r}^{p, q} \rightarrow V_{r}^{p-r, q+r-1}\right)}{\operatorname{Im}\left(\delta_{r}: V_{r}^{p+r, q-r+1} \rightarrow V_{r}^{p, q}\right)} .
$$

In particular, we have $V_{0}^{p, q}=C_{p+q-p \Sigma_{L}}^{f}, V_{1}^{p, q}=H_{p+q-p \Sigma_{L}}\left(L ; \mathbb{Z}_{2}\right), \delta_{1}=\left[\partial_{1}\right]$.

(4) $E_{r}^{p, q}$ collapses to $E_{v+1}^{p, q}=E_{v+2}^{p, q}=\cdots=E_{\infty}^{p, q}$ at $(v+1)$-step and for each $p \in \mathbb{Z}$ it holds

$$
\bigoplus_{q \in \mathbb{Z}} E_{\infty}^{p, q} \cong H F(L) \otimes_{\mathbb{Z}_{2}} A
$$

where we know that $v=\left[\frac{\operatorname{dim} L+1}{\Sigma_{L}}\right]$. 
Damian ([3]) provided the theory of the lifted Floer homology $H F^{\bar{L}}(L)$ for an arbitrary covering $\bar{L} \rightarrow L$. Let $p: \bar{L} \rightarrow L$ be a covering map of a compact Lagrangian submanifold $L$ embedded in $M$. We need to assume that $L$ is monotone with $N_{L} \geq 3$. By lifting to the covering space $\bar{L}$ all data on $L$ necessary to define the Floer homology $H F(L)$, Damian defined the lifted Floer complex $C F^{\bar{L}}$ and the lifted Floer homology $H F^{\bar{L}}(L)$ of $L$. The Hamiltonian invariance of the lifted Floer homology also holds. Moreover he constructed the spectral sequence converging to $H F^{\bar{L}}(L)$ with the Morse homology of $\bar{L}$ as the first step. Note that the lifted Floer homology $H F^{\bar{L}}(L)$ is not well-defined in the case of $\Sigma_{L}=2$. See [3] for the details. By definition the non-vanishing of the lifted Floer homology $H F^{\bar{L}}(L)$ also implies the Hamiltonian non-displaceability of $L$. However there seems to be no direct relationship between the original Floer homology and the lifted Floer homology.

\section{Floer homology and lifted Floer homology of Gauss images of isoparametric hypersurfaces}

Suppose that $L^{n}=\mathscr{G}\left(N^{n}\right) \subset Q_{n}(\mathbb{C})$ is the Guass image of an isoparametric hypersurface $N^{n}$ in $S^{n+1}$ with $g$ distinct principal curvatures and multiplicities $\left(m_{1}, m_{2}\right)$. Since it follows from Theorem 1 (3) that $v=\left[\frac{\operatorname{dim} L+1}{\Sigma_{L}}\right]=\left[\frac{(n+1) g}{2 n}\right]$, we get

Lemma 1 ([11]). For each $p, q \in \mathbb{Z}$ it holds

(0) $E_{1}^{p, q}=E_{\infty}^{p, q}(v=0)$ if and only if $g=1$ and $n \geq 2$.

(1) $E_{2}^{p, q}=E_{\infty}^{p, q}(v=1)$ if and only if $(g, n)=(1,1), g=2$ or $\left(g, m_{1}, m_{2}\right)=$ $(3,2,2),(3,4,4),(3,8,8)$.

(2) $E_{3}^{p, q}=E_{\infty}^{p, q}(v=2)$ if and only if $\left(g, m_{1}, m_{2}\right)=(3,1,1)$ or $g=4$.

(3) $E_{4}^{p, q}=E_{\infty}^{p, q}(v=3)$ if and only if $\left(g, m_{1}, m_{2}\right)=(6,1,1)$ or $(6,2,2)$.

In the case when $g=1$ or $g=2$, since the Gauss image of isoparametric hypersurfaces are nothing but real forms of complex hyperquadrics, it is well-known that $H F(L) \cong H_{*}\left(L ; \mathbb{Z}_{2}\right)$ ([19], [10]).

In the case when $g=3$, that is, $N^{n}$ is a Cartan hypersurface, we proved

Lemma 2 ([11]). The Gauss image of $L^{n}=\mathscr{G}\left(N^{n}\right)$ of each isoparametric hypersurface with $g=3$ is a $\mathbb{Z}_{2}$-homology sphere $\left(\right.$ i.e. $H_{k}\left(L^{n} ; \mathbb{Z}_{2}\right)=0$ for each $0<k<n$ ) satisfying $H_{1}\left(L^{n} ; \mathbb{Z}\right) \cong \mathbb{Z}_{3}$.

The Gauss images of Cartan hypersurfaces provide new examples of Lagrangian $\mathbb{Z}_{2}$-homology spheres embedded in compact Hermitian symmetric spaces.

This result is quite essential for the proof of main theorem [11] in the case when $g=3$. When $g=3$ and $m=m_{1}=m_{2}=2,4$ or 8 , by Lemma 1 we have $v=1$. By Lemma 2 the spectral sequence

$$
\left[\partial_{1}\right]: H_{p+q-2 m p}\left(L ; \mathbb{Z}_{2}\right) \rightarrow H_{p+q-2 m p+2 m-1}\left(L ; \mathbb{Z}_{2}\right),
$$


namely

$$
\left[\partial_{1}\right]: H_{k}\left(L ; \mathbb{Z}_{2}\right) \rightarrow H_{k+2 m-1}\left(L ; \mathbb{Z}_{2}\right) \quad(k=0,1, \cdots, n)
$$

implies $\left[\partial_{1}\right]=0$, because we see that, $H_{k}\left(L ; \mathbb{Z}_{2}\right)=\{0\}$ or $H_{k+2 m-1}\left(L ; \mathbb{Z}_{2}\right)=\{0\}$ since $L$ is a $\mathbb{Z}_{2}$-homology sphere. Thus $d_{1}=0$. The spectral sequence becomes

$$
V_{2}^{p, q}=\frac{\operatorname{Ker}\left(\left[\partial_{1}\right]: V_{1}^{p, q} \rightarrow V_{1}^{p-1, q}\right)}{\operatorname{Im}\left(\left[\partial_{1}\right]: V_{1}^{p+1, q} \rightarrow V_{1}^{p, q}\right)}=V_{1}^{p, q}=H_{p+q-p \Sigma_{L}}\left(L ; \mathbb{Z}_{2}\right) .
$$

and $E_{\infty}^{p, q}=E_{2}^{p, q}=V_{1}^{p, q} \otimes A^{p \Sigma_{L}} \cong H_{p+q-p \Sigma_{L}}\left(L ; \mathbb{Z}_{2}\right) \otimes A^{p \Sigma_{L}}$. Hence we obtain

$$
H F(L) \cong \bigoplus_{q \in \mathbb{Z}} E_{\infty}^{p, q}=\bigoplus_{q \in \mathbb{Z}} E_{2}^{p, q} \cong \bigoplus_{q \in \mathbb{Z}} H_{p+q-p \Sigma_{L}}\left(L ; \mathbb{Z}_{2}\right) \otimes A^{p \Sigma_{L}} \cong H_{*}\left(L ; \mathbb{Z}_{2}\right) .
$$

Concerned with the lifted Floer homology to $\mathscr{G}: N \rightarrow L=\mathscr{G}(N)$, similarly using Damian's spectral sequence and the homological data of isoparametric hypersurfaces $N$ ([16]) we obtain $H F^{N}(L) \cong H_{*}\left(N ; \mathbb{Z}_{2}\right)$.

Theorem 4 (IMMO [11]). In the case when $g=3$ and $m=m_{1}=m_{2}=2,4$ or 8 , the Floer homology $H F(L)$ (resp. the lifted Floer homology $H F^{N}(L)$ ) is isomorphic to $H_{*}\left(L ; \mathbb{Z}_{2}\right)\left(\operatorname{resp} . H_{*}\left(N ; \mathbb{Z}_{2}\right)\right)$.

In particular, $H F(L) \neq\{0\}$ and thus we see that for any $\phi \in \operatorname{Haml}\left(Q_{n}(\mathbb{C}), \omega_{\text {std }}\right)$ with $L \pitchfork \phi(L)$, it holds $\sharp(L \cap \phi(L)) \geq \operatorname{rank} H_{*}\left(L^{n} ; \mathbb{Z}_{2}\right)=2$.

In the case when $g=4$ or 6 , we use homological data on isoparemetric hypersurfaces $N^{n}$ ([16]) and the spectral sequence for the lifted Floer homology $H F^{L}(L)$ applied to the covering map $\mathscr{G}: \bar{L}=N \rightarrow L=\mathscr{G}(N)$ (Damian [3]) in order to discuss the non-vanishing of the lifted Floer homology.

Theorem 5 (IMMO [11]). In the case when $g=4$ or $g=6$ except for the remaining three cases as below, the lifted Floer homology $H F^{N}(L)$ is non-zero:

$$
\begin{array}{ll}
\left(g, n, m_{1}, m_{2}\right)=(3,3,1,1), & N=\frac{S O(3)}{\mathbb{Z}_{2}+\mathbb{Z}_{2}}, \\
\left(g, n, m_{1}, m_{2}\right)=(4,2 k+2,1, k), & N=\frac{S O(2) \times S O(k+2)}{\mathbb{Z}_{2} \times S O(k)}(k \geq 1), \\
\left(g, n, m_{1}, m_{2}\right)=(6,6,1,1), & N=\frac{S O(4)}{\mathbb{Z}_{2}+\mathbb{Z}_{2}},
\end{array}
$$

Notice that $\left(g, n, m_{1}, m_{2}\right)=(1,1,1,-),(2,2,1,1),(3,3,1,1),(4,4,1,1)$ or $(6,6,1,1)$ if and only if the minimal Maslov number of the Gauss image $L$ of isoparametric hypersurface has $\Sigma_{L}=2$, then any lifted Floer homology $H F^{\bar{L}}(L)$ is not well-defined.

Problem 1. Determine whether the lifted Floer homology $H F^{\bar{L}}(L)$ is nonzero or not in the case when $\left(g, n, m_{1}, m_{2}\right)=(4,2 k+2,1, k)(k \geq 2)\left(\right.$ then $\left.\Sigma_{L}=k+1 \geq 3\right)$.

Problem 2. Determine whether the Floer homology $H F(L)$ is nonzero or not in the case when $\left(g, n, m_{1}, m_{2}\right)=(3,3,1,1),(4,4,1,1)$ or $(6,6,1,1)$ (then $\left.\Sigma_{L}=2\right)$. When is the Floer homology $H F(L)$ isomorphic to $H_{*}\left(L ; \mathbb{Z}_{2}\right)$ ?

More generally we should pose the following problem as our goal: 
Problem 3. Determine explicitly the Floer homology $H F(L)$ of the Gauss images of isoparametric hypersurfaces in the case when $(g, m)=(3,1), g=4$ or $g=6$.

Since the Floer homology is based on the Mores homology, it is quite natural to study the following problems:

Problem 4. Determine explicitly the homology $H F_{*}\left(L ; \mathbb{Z}_{2}\right)$ of the Gauss images of isoparametric hypersurfaces in the case when $g=4$ or $g=6$.

Problem 5. Construct concretely the Morse homology of the Gauss images of isoparametric hypersurfaces in the case when $g=3,4$ or $g=6$.

Acknowledgements The author sincerely would like to thank Professor Young Jin Suh (Director of RIRCM) for his leading the international joint project of submanifold theory in differential geometry and his organization of twenty times successful workshops. The author also would like to thank Dr. Hyunjin Lee for her excellent supports to those workshops. The author was partly supported by JSPS Grant-in-Aid for Scientific Research (C) No. 15K04851 and JSPS Program for Advancing Strategic International Networks to Accelerate the Circulation of Talented Researchers "Mathematical Science of Symmetry, Topology and Moduli, Evolution of International Research Network based on OCAMI “.

\section{References}

1. P. Biran, Lagrangian non-intersections, Geom. Funct. Anal. 16 (2006), 279-326.

2. Bott, Raoul; Tu, Loring W. Differential forms in algebraic topology. Graduate Texts in Mathematics, 82. Springer-Verlag, New York-Berlin, 1982. xiv+331 pp. ISBN: 0-387-90613-4

3. M. Damian, Floer homology on the universal cover, Audin's conjecture and other constraints on Lagrangian submanifolds, Comment. Math. Helv. 87 (2012), 433-462.

4. A. Floer, Morse theory for Lagrangian intersections. J. Differential Geom. 28 (1988), 513547.

5. A. Floer, A relative Morse index for the symplectic action. Comm. Pure Appl. Math. 41 (1988), 393-407.

6. A. Floer, The unregularized gradient flow of the symplectic action. Comm. Pure Appl. Math. 41 (1988), 774-813.

7. A. Floer, Witten's complex and infinite dimensional Morse theory. J. Differential Geom. 30 (1989), 207-221.

8. M. Gromov, Pseudo holomorphic curves in symplectic manifolds, Invent. Math. 82 (1985), 307-347.

9. H. Iriyeh, Symplectic topology of Lagrangian submanifolds of $\mathbb{C} P^{n}$ with intermediate minimal Maslov numbers, to appear in Advances in Geometry. (Preprimt, arXiv:1401.0777v1,2014.)

10. H. Iriyeh, T. Sakai and H. Tasaki, Lagrangian Floer homology of a pair of real forms in Hermitian symmetric spaces of compact type. J. Math. Soc. Japan 65 (2013), 1135-1151.

11. H. Iriyeh, H. Ma, R. Miyaoka and Y. Ohnita, Hamiltonian non-diaplaceability of Guass images of isoparametric hypersurfaces, Bulletin of the London Mathematical Society 2016; doi: 10.1112/blms/bdw040

12. H. Ma and Y. Ohnita, On Lagrangian submanifolds in complex hyperquadrics and isoparametric hypersurfaces in spheres, Math. Z. 261 (2009), 749-785.

13. H. Ma and Y. Ohnita, Differential geometry of Lagrangian submanifolds and Hamiltonian variational problems, Harmonic maps and differential geometry, Contemp. Math., 542, 115134 Amer. Math. Soc., Providence, RI, 2011. 
14. H. Ma and Y. Ohnita, Hamiltonian stability of the Gauss images of homogeneous isoparametric hypersurfaces. I, J. Differential Geom. 97 (2014), 275-348.

15. H. Ma and Y. Ohnita, Hamiltonian stability of the Gauss images of homogeneous isoparametric hypersurfaces. II, Tohoku Math. J. 67 (2015), 195-246

16. H. F. Münzner, Isoparametrische Hyperfläche in Sphären. Math. Ann. 251 (1980), 57-71. Isoparametrische Hyperfläche in Sphären, II. Math. Ann. 256 (1981), 215-232.

17. Y.-G. Oh, Floer cohomology of Lagrangian intersections and pseudo-holomorphic disks, I, Comm. Pure Appl. Math. 46 (1993), 949-994.

18. Y.-G. Oh, Addendum to "Floer cohomology of Lagrangian intersections and pseudoholomorphic disks, I”, Comm. Pure Appl. Math. 48 (1995), 1299-1302.

19. Y.-G. Oh, Floer cohomology of Lagrangian intersections and pseudo-holomorphic discs III, H. Hofer, et al. (Eds.), The Floer Memorial Volume, Birkhäuser, Basel, 1995, 555-573.

20. Y.-G. Oh, Floer cohomology, spectral sequences, and the Maslov class of Lagrangian embeddings, Int. Math. Res. Not. 7 (1996), 305-346.

21. Y. Ohnita, Geometry of Lagrangian Submanifolds and Isoparametric Hypersurfaces, Proceedings of the 14th International Workshop on Differential Geometry and the 3rd KNUGRGOCAMI Differential Geometry Workshop, Volume 14, 43-67, Natl. Inst. Math. Sci. (NIMS), Taejeon 2010. (OCAMI Preprint Ser. no.10-9.)

22. H. Ono, Integral formula of Maslov index and its applications, Japan. J. Math. 30 (2004), 413-421.

23. B. Palmer, Hamiltonian minimality and Hamiltonian stability of Gauss maps, Diff. Geom. and its Appl. 7 (1997), 51-58.

24. C. Viterbo, Intersection de sous-variétés Lagrangiennes, fonctionelles d'action et indice des systèmes Hamiltoniens, Bull. Soc. Math. France 115 (1987), 361-390. 